\title{
Qualität und persönlicher Einsatz: Wachstum in der Berg- und Expeditionsmedizin
}

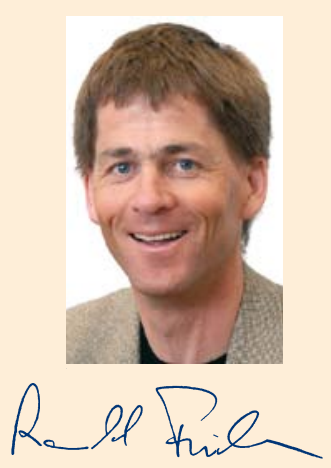

Ihr

PD Dr. Rainald Fischer, München
Liebe Kolleginnen und Kollegen,

in Zeiten, in denen weltweit vieles auf Stagnation oder Rückgang hinweist - man denke nur an die globale - ist es erfreulich, dass zumindest manche Bereiche stabile Wachstumszahlen aufweisen.

Wir veranstalteten Mitte Juli den 100. Alpinärztekurs im 20. Lehrgangsjahr mit über 4000 Teilnehmern. Damit sind die Kurse der Österreichischen Gesellschaft für Alpin- und Höhenmedizin (www. alpinaerzte.org) und der Deutschen Gesellschaft für Berg- und Expeditionsmedizin (www.bexmed.de) die erfolgreichsten Kurse für Alpinärzte weltweit.

Warum sind diese Kurse so erfolgreich, obwohl es sich doch um ein Randgebiet der Medizin handelt?

Meiner Meinung nach sind dafür mehrere Gründe verantwortlich:

- Kontinuität von Beginn an: Seit 1992 hat sich Prof. Franz Berghold, Kaprun, unermüdlich und konsequent um die Gestaltung bemüht. Auch heute noch betreut er jeden Sommerkurs und die meisten Winterkurse. Das gewährleistet gleichbleibende Qualität und optimalen Ablauf.

- Qualität: Ein umfangreiches Lehrskriptum erscheint mittlerweile in der 7. Auflage. Inhaltlich ist es vergleichbar mit anerkannten Lehrbüchern der Alpinmedizin - referiert wird es von bewährten deutschen und österreichischen Experten, die in ihren Fachgebieten sicherlich zu den Spezialisten zählen. Kursteilnehmer erhalten so das gesamte Spektrum der Alpin- und Höhenmedizin vermittelt, das nicht nur aus Theorie besteht: Praktische Fähigkeiten und Kenntnisse im Bergsport und bei der Bergrettung gehören dazu. Auch Neulinge können an den Lehrgangsorten Adamekhütte, FranzSenn-Hütte und Planneralm grundlegende Kenntnisse erwerben. Erfahrene Bergsteiger können sie mit Bergführern steigern und ausbauen.
- Internationale Anerkennung: Die beiden Gesellschaften bieten inzwischen das gesamte mögliche Spektrum in der Bergrettungsmedizin an. Die Alpinärztekurse führen in einem dreiwöchigem Curriculum (Frühjahr-, Sommerund Winterkurs) zum international anerkannten Doctor of Mountain Medicine. Die Anerkennung durch die Medizinische Kommission der UIAA (internationale Vereinigung der nationalen Alpenvereine) und der Medizinischen Kommission der IKAR (internationale Bergrettungsorganisation) gibt es bereits seit 1997. Ein Jahr später wurde das Curriculum auch von der International Society of Mountain Medicine (ISMM) anerkannt und seither regelmäßig verlängert. Deshalb können Kursteilnehmer mit einer Prüfung das international gültige Diploma of Mountain Medicine erwerben. Zusätzliche Kurse für das Diploma of Expedition and Wilderness Medicine und das Diploma of Mountain Rescue Medicine sind ebenfalls möglich.

- Kooperation: Im Gegensatz zu vielen Gebieten der Medizin, wo Konkurrenz an der Tagesordnung ist, arbeiten die deutsche und die österreichische Gesellschaft für Bergmedizin seit Jahren harmonisch miteinander. In gemeinsamen Ausschüssen legen wir das Lehrgangskonzept fest und führen die Prüfungen jährlich abwechselnd durch. Die Referenten sind Mitglieder aus beiden Gesellschaften. Entsprechend steht es auch jedem Teilnehmer frei, ob er in der Deutschen Gesellschaft für Berg- und Expeditionsmedizin oder der Österreichischen Gesellschaft für Alpin- und Höhenmedizin Mitglied sein möchte. Ich präferiere natürlich eine Mitgliedschaft in der BExMed.

Diese und noch eine Menge anderer Gründe machen die Kurse so attraktiv. Eine Teilnahme kann ich allen Lesern der FTR nur empfehlen. Ich wünsche mir, dass ich an dieser Stelle schon in 10 Jahren zum 200. Kurs gratulieren kann. 